\title{
Cross-linked chitosan-glyoxal/kaolin clay composite: Parametric optimization for color removal and COD reduction of remazol brilliant blue $\mathrm{R}$ dye
}

\author{
Ali H. Jawad ( $\sim$ ahjm72@gmail.com ) \\ Universiti Teknologi MARA https://orcid.org/0000-0002-4827-9093 \\ Ahmed Saud Abdulhameed \\ University of Baghdad \\ Elmira Kashi \\ Universiti Teknologi MARA \\ Zaher Mundher Yaseen \\ Ton Duc Thang University \\ Zeid A. ALOthman \\ King Saud University \\ Mohammad Rizwan Khan \\ King Saud University
}

\section{Research Article}

Keywords: Chitosan, Kaolin, COD reduction, Glyoxal, Remazol brilliant blue R, Box-Behnken design

Posted Date: April 21st, 2021

DOl: https://doi.org/10.21203/rs.3.rs-438826/v1

License: (c) (i) This work is licensed under a Creative Commons Attribution 4.0 International License.

Read Full License

Version of Record: A version of this preprint was published at Journal of Polymers and the Environment on May 28th, 2021. See the published version at https://doi.org/10.1007/s10924-021-02188-1. 
Cross-linked chitosan-glyoxal/kaolin clay composite: Parametric optimization for color removal and COD reduction of remazol brilliant blue $\mathrm{R}$ dye

Ali H. Jawad ${ }^{\mathrm{a},}$, , Ahmed Saud Abdulhameed ${ }^{\mathrm{b}}$, Elmira Kashi ${ }^{\mathrm{a}}$, Zaher Mundher Yaseen ${ }^{\mathrm{c}}$, Zeid A. ALOthman $^{\mathrm{d}}$, Mohammad Rizwan Khan ${ }^{\mathrm{d}}$

${ }^{a}$ Faculty of Applied Sciences, Universiti Teknologi MARA, 40450 Shah Alam, Selangor, Malaysia

${ }^{\mathrm{b}}$ Department of Medical Instrumentation Engineering, Al-Mansour University College, Baghdad, Iraq

${ }^{c} \mathrm{New}$ era and development in civil engineering research group, Scientific Research Center, AlAyen University, Thi-Qar, 64001, Iraq.

${ }^{\mathrm{d}}$ Chemistry Department, College of Science, King Saud University, Riyadh 11451, Saudi Arabia

Corresponding author:

E-mail: ali288@uitm.edu.my; ahjm72@gmail.com (Ali H. Jawad) 


\begin{abstract}
Kaolin clay $(\mathrm{KN})$ was employed as an inorganic filler to modify a cross-linked chitosan-glyoxal as Schiff's-based chitosan composite derivative (CTS-GLY). The resulting (CTS-GLY/KN) was found to be a promising composite synthetic biopolymer that can be potentially utilized for color removal as well as COD reduction of an industrial anionic dye (remazol brilliant blue R, RBBR). The surface porosity, crystallinity, morphology, functionality, charge, and amine content of the CTS-GLY/KN were studied using BET, XRD, SEM, FTIR, $\mathrm{pH}_{\mathrm{pzc}}$ and $\mathrm{pH}$-potentiometric titration analyses, respectively. Response surface methodologyBox-Behnken design (RSM-BBD) was used to optimize the impact of the main input factors on the color removal and COD reduction of RBBR. The adsorptive performance CTS-GLY/KN towards RBBR was well-defined by both Langmuir and Freundlich isotherm models with highest adsorption capacity of $447.1 \mathrm{mg} / \mathrm{g}$ at $30{ }^{\circ} \mathrm{C}$. This finding reveals that $\mathrm{CTS}-\mathrm{GLY} / \mathrm{KN}$ can be utilized as a promising, feasible, and environmentally friendly composite-biosorbent for color removal and COD reduction of textile dyes from aqueous medium.
\end{abstract}

Keywords: Chitosan; Kaolin; COD reduction; Glyoxal; Remazol brilliant blue R; Box-Behnken design 


\section{Introduction}

The considerable usage of organic dyes and pollutants in numerous industries such as textiles, pharmaceuticals, rubber, and paper generate a vast number of effluents containing dyes [1]. The release of the effluents into water bodies causes significant variations to appear in water attributes including color, $\mathrm{pH}$, and chemical oxygen demand (COD), as well as detrimental effects on human health [2]. Severe dye residues are one of the significant attributes of textile wastewater, which can prevent sunlight penetration into water bodies resulting in reduction in photosynthetic activities of aquatic life [3]. One of the most frequent pollutants is COD, indicating the existence of organic compounds in wastewater [4]. To avoid risking the survival of aquatic life by exhausting the oxygen resources, it is essential to reduce COD levels and meet the allowed discharge threshold [5]. Remazol Brilliant Blue R (RBBR) dye is a reactive dye commonly used as an initial ingredient for generating polymeric dyes in textiles [6].

RBBR considers as a good model for toxic and recalcitrant organic pollutants that poses a threat to eco-system and humans [7]. Accordingly, the dye removal and COD reduction from wastewater prior to release into the water bodies is a necessary action to maintain both the humans and the environment healthy. Conventional wastewater treatment methods such as adsorption [8], nanofiltration [9], and ozonation [10] have been utilized for the removal of organic dyes and to attain reduction in COD levels of the wastewater. Simple operation design, non-sensitivity to pollutants, recyclability, being highly efficient, cost-effective, and lesser waste residuals has made adsorption among the most employed methods [11].

Chitosan (CTS) is a cationic polysaccharide of $\beta$-(1-4) acetyl-D-glucosamine units and derived from deacetylating chitin, found in the exoskeleton of crustaceans, insects etc [12, 13]. CTS reserves excellent properties, such as biodegradability, hydrophilicity, high chemical 
reactivity, biocompatibility, chirality, adsorption capacity, non-toxicity, anti-bacterial activity, and chelation $[14,15]$. CTS is featured in the existence of amino $\left(-\mathrm{NH}_{2}\right)$ and hydroxyl $(-\mathrm{OH})$ groups in its polymeric chain, acting as the prospect adsorption sites for removal of metal ions and anionic dyes $[16,17]$. However, natural CTS is sensitive to $\mathrm{pH}$ changes, mechanically unstable, susceptible to swelling, and leachable in aqueous environment [18]. Hence, it is of great significance to improve the physiochemical properties of CTS.

Many approaches have been carried out to improve the physicochemical properties of CTS such as crosslinking reaction [19], grafting with amino functional groups [20], and compositing with nanomaterials [21]. Amongst the approaches, crosslinking reaction is the most convenient pathway for developing attributes of CTS such as its mechanical strength, solubility in acidic medium, chemical stability and hydrophobicity [22, 23]. Many studies have been carried out on utilizing crosslinked CTS derivatives as adsorbents for removal of dyes from aqueous solutions $[24,25]$. Furthermore, CTS-based composite is considered as one of the most desired approaches for improving the surface and structural properties of CTS [26]. Consequently, several decent fillers have been introduced for preparation of CTS-based composite biopolymer to enhance physiochemical and adsorptive properties. These fillers include clay [27], metal oxide nanoparticles [28], semiconductor [22], montmorillonite [29, 30], carbon nanotubes [31], and silica [32].

Kaolin clay $(\mathrm{KN})$ is one of the most common natural inorganic clays, existing in rocks copiously in crystalline structure [33]. KN has outstanding properties (e.g., porosity, low-cost, thermal stability, abundant availability, and non-toxicity) in environmental applications when employed as adsorbent alone and/or within a CTS composite, such as good adsorption capacity, high surface area, easy to functionalization its surface, high thermal stability, and 
environmentally friendly [34]. Recent studies show that CTS and KN complement each other for producing multifunctional biocomposite adsorbents, which can be effectively employed for removal of numerous water contaminants including organic anionic dyes [27], biological contaminants [35], organic cationic dyes [36], and nutrients [37]. From the environmental standpoint, utilizing kaolin clay powder as a filler or surface modifier to chitosan will improve the surface area and adsorptive property of chitosan, and will reduce the cost and environmental impact of solid waste disposal. Hence, these previous outstanding applications inspire us to introduce Schiff's base-crosslinked chitosan-glyoxal/kaolin (CTS-GLY/KN) composite as a new biocomposite material with multifunctional performances for color removal (CR) and reduction of chemical oxygen demand (COD) of an industrial anionic dye (Remazol Brilliant Blue R) from contaminated water. Statistical optimization by Box-Behnken design (BBD) is gathered to obtain maximum outputs from minimum inputs. Furthermore, the adsorption mechanism, isotherms, and kinetics of RBBR were investigated as well.

\section{Materials and methods}

\subsection{Materials}

Medium MW Chitosan (CTS) with deacetylation degree of $75 \%$, Kaolin clay (KN), glyoxal (GLY) solution, and RBBR (MW: 626; g/mol $\lambda_{\max }=592 \mathrm{~nm}$ ) were acquired from Sigma-Aldrich. Ultrapure water $(18.2 \mathrm{M} \Omega / \mathrm{cm})$ was utilized to prepare all the reagents and solutions. 


\subsection{Synthesis of CTS-GLY/KN}

CTS-GLY/KN composite was prepared according to the published method [18]. In this preparation, the CTS-GLY/KN was obtained by combining $1 \mathrm{~g}$ CTS flakes and $1 \mathrm{~g} \mathrm{KN}$ powder (50\% CTS: $50 \% \mathrm{KN}$, this ratio was predetermined to be the best) in a beaker holding $60 \mathrm{~mL}$ of acetic acid solution with 5\% v/v concentration while being stirred strenuously for the duration of $24 \mathrm{~h}$ at room temperature to dissolve the CTS flakes. CTS/KN beads were obtained by injecting droplets of CTS/KN viscous solution into a beaker containing $1 \mathrm{~L}$ of $\mathrm{NaOH}$ solution $(0.5 \mathrm{M})$ by a syringe needle $(10 \mathrm{~mL})$. Afterwards, deionized water was utilized to wash the remaining sodium hydroxide from the CTS/KN beads. The Schiff's base formation was achieved by cross-linking reaction with $2 \%$ GLY according to the published procedure [38]. After that, the composite (CTS-GLY/KN) beads were washed using deionized water before being dried at room temperature for $24 \mathrm{~h}$. Then, the CTS-GLY/KN beads were ground into fine particles and left to dry inside an oven for $24 \mathrm{~h}$ at $80{ }^{\circ} \mathrm{C}$. Eventually, the CTS-GLY/KN in powder form was sieved to attain a final particle size $\leq 250 \mu \mathrm{m}$. The steps taken to synthesize CTS-GLY/KN are illustrated in Fig.1.

\subsection{Characterization}

The surface porosity, surface morphology and surface functional groups of CTSGLY/KN before and after RBBR uptake were analyzed utilizing Micromeritics ASAP 2060 analyzer (by measuring $\mathrm{N}_{2}$ adsorption/desorption isotherms at $77 \mathrm{~K}$ ), a Scanning electron microscopy-energy dispersive X-ray (SEM-EDX, TM3030Plus, Tabletop Microscope, Hitachi, Japan) and a Fourier transform infrared (FTIR) spectrophotometer at range 450-4000 cm-1 (Perkin-Elmer, Spectrum RX I), respectively. Crystalline structure of CTS-GLY/KN was studied using X-ray diffractometer (XRD, $\mathrm{Cu} \mathrm{K} \alpha$ radiation $\lambda=1.54 \AA$, X'Pert PRO, PAnalytical) and 
the zero point of charge (pHpzc) of the CTS-GLY/KN was arbitrated with reference to the methodology illustrated in the literature [39]. The unreacted amino $\left(-\mathrm{NH}_{2}\right)$ groups present in the polymeric matrix composite were estimated by $\mathrm{pH}-$ potentiometric titration approach [40].

\subsection{Experimental design}

The effects of three adsorption parameters (CTS-GLY/KN dose, contact time and $\mathrm{pH}$ of treatment environment) on the $\mathrm{CR}$ and COD reduction of RBBR were studied and optimized using RSM-BBD method. The Stat-Ease Design Expert (version 13.0, Minneapolis, USA) software was utilized for the purpose of designing the adsorption experiments, optimizing the processes, and finally statistically analyzing the data. The levels and coded values of the independent parameters used for designing the experiments are displayed in Table 1. The following quadratic equation (1) was employed to analyze the experimental data and predict the CR and COD values:

$$
Y=\beta_{0}+\sum \beta_{i} X_{i}+\sum \beta_{i i} X_{i}^{2}+\sum \sum \beta_{i j} X_{i} X_{j}
$$

where, $Y$ represents the predicted response (RBBR color removal (\%), and COD reduction (\%)); $X_{i}$ and $X_{j}$ are coded the independent variables; $-\beta_{0}$ is the constant; $\beta_{i}, \beta_{i i}$ and $\beta_{i j}$ are coefficients of linear, quadratic, and interactive coefficient of input independent variables, respectively. To investigate and optimize the effect of the three parameters (CTS-GLY/KN dose, time, and $\mathrm{pH}$ ) on CR value and COD reduction by CTS-GLY/KN, BBD opted 17 experimental runs consisting of three factors with three value levels and five center points: A: CTS-GLY/KN dose $(0.02-0.1$ g), B: $\mathrm{pH}(4-10)$, and $\mathrm{C}$ : time (5-30 min). Table 2 presents the BBD matrix and obtained response results of $\mathrm{CR}$ and COD reduction of RBBR. Specific amount of the adsorbent was 
poured into Erlenmeyer flasks $(250 \mathrm{~mL})$ containing $100 \mathrm{~mL}$ of RBBR solution then placed inside a thermostat water bath shaker. Agitation of the solutions was achieved by shaking at a constant speed of 100 strokes per minute. Then, the adsorbents were separated from the solution after adsorption process using a syringe filter $(0.45 \mathrm{~lm})$. UV-Vis spectrophotometer (HACH DR 3900) was utilized to measure the RBBR CR and COD concentrations at the wavelength of maximum absorption $\left(\lambda_{\max }\right) 592 \mathrm{~nm}$ and $620 \mathrm{~nm}$, respectively. Eq. 2 and Eq. 3 were adopted to calculate the $\mathrm{CR} \%$ and COD \% of RBBR, respectively.

$C R \%=\frac{\left(C_{o}-C_{e}\right)}{C_{o}} \times 100$

$\operatorname{COD} \%=\frac{\left(D_{o}-D_{e}\right)}{D_{o}} \times 100$

Where $C_{o}(\mathrm{mg} / \mathrm{L})$ and $C_{e}(\mathrm{mg} / \mathrm{L})$ are RBBR concentrations at the beginning and at equilibrium, respectively while $\mathrm{D}_{o}(\mathrm{mg} / \mathrm{L})$ and $D_{e}(\mathrm{mg} / \mathrm{L})$ are concentrations of COD at the beginning and at equilibrium, respectively.

\subsection{Adsorption study of RBBR on CTS-GLY/KN}

The study on the RBBR uptake by CTS-GLY/KN was done in batch mode. The obtained results are presented Table 2 which shows the highest color removal (97.3\%) and COD reduction (81.8\%) were achieved in the following experimental conditions: time (30 min), solution $\mathrm{pH}(4)$, and CTS-GLY/KN dose $(0.06 \mathrm{~g})$. These conditions were later applied to test the adsorption values of RBBR at differing initial concentrations $(50-400 \mathrm{mg} / \mathrm{L})$ of the dye and contact time (0- 
$360 \mathrm{~min}$ ) using identical batch experiment process as abovementioned. Eq. 4 was used for calculating the adsorption capacity of CTS-GLY/K toward RBBR at equilibrium $q_{e}(\mathrm{mg} / \mathrm{g})$.

$q_{e}=\frac{\left(C_{o}-C_{e}\right) V}{W}$

where, $V(\mathrm{~L})$ is the volume of RBBR solution, while $W(\mathrm{~g})$ represents the weight of CTSGLY/KN composite used.

\section{Results and Discussion}

\subsection{Characterization of CTS-GLY/KN}

The physicochemical characteristics of CTS-GLY/KN are presented in Table 3. The free amine groups (\%) of the CTS-GLY/KN, that are considered as one of the most active adsorption sites in the CTS composites for adsorption of anionic dyes, are determined through $\mathrm{pH}$ potentiometric titration. The results demonstrate a satisfactory value of $27.4 \%$ free amine groups (\%) in the CTS-GLY/KN composite, suggesting a good potential for adsorbing the RBBR molecules. It was found from the BET analysis that the surface area of CTS-GLY/KN is $39.8\left(\mathrm{~m}^{2} / \mathrm{g}\right)$, while mean pore diameter is $6.2 \mathrm{~nm}$. This result indicates a higher surface area for CTS-GLY/KN than that of the CTS-GLY $\left(0.39 \mathrm{~m}^{2} / \mathrm{g}\right)$ as reported in a previous study [38], which can be attributed to the fact that the KN particles have a high surface area, scattered on the CTSGLY matrix, which enlarges its surface [41]. Similar observations have been reported in our previous studies for the improvement the surface area of the crosslinked chitosan by intercalation of the KN clay in its structure [41, 42]. According to the IUPAC classification, the CTSGLY/KN is a mesoporous material, with a mean pore diameter $(2-50 \mathrm{~nm})$ greater than that of the RBBR molecules $(1.6 \mathrm{~nm})$ [43]. This result indicates that the pores of CTS-GLY/KN can easily accommodate the RBBR molecules. 
The X-Ray Diffraction pattern of the CTS-GLY/KN is illustrated in Fig. 2, exhibiting sharp characteristic diffraction peaks at $2 \theta=26,38^{\circ}, 44^{\circ}, 64^{\circ}$, and $78^{\circ}$ corresponding to the kaolinite $\mathrm{Al}_{2} \mathrm{Si}_{2} \mathrm{O}_{5}(\mathrm{OH})_{4}$ [44]. These peaks reveal the high crystallinity of CTS-GLY/KN caused by the strong electronic interactions between the positively charged of crosslinked biopolymer surface (CTS-GLY) and negatively charge of KN clay, evidencing intercalation of KN particles into the structure of molecules of CTS-GLY/KN $[45,46]$.

FTIR spectral analysis was performed to identify the changes in the functional groups of the CTS-GLY/KN before and after adsorption of RBBR as shown in Fig. 3a and Fig. 3b, respectively. The FTIR spectrum of CTS-GLY/KN (Fig. 3a) displays the characteristic peaks at $3400 \mathrm{~cm}^{-1}, 1640 \mathrm{~cm}^{-1}$, and $1049 \mathrm{~cm}^{-1}$, that can be related to vibrations of $-\mathrm{OH}$ and $-\mathrm{NH}_{2}$, vibration of $\mathrm{C}=\mathrm{N}$, and stretching vibration of $\mathrm{Si}-\mathrm{O}-\mathrm{Si}[34,38]$. The bands at $2900-2860 \mathrm{~cm}^{-1}$, $1375 \mathrm{~cm}^{-1}, 940 \mathrm{~cm}^{-1}$, and $500-795 \mathrm{~cm}^{-1}$ are due to the stretching vibrations of C-H symmetrical and asymmetrical, stretching vibration of $\mathrm{C}-\mathrm{N}$, bending vibration of $-\mathrm{OH}$ in $\mathrm{Al}-\mathrm{OH}$, and vibrations of Si-O-Al, respectively [47]. The FTIR spectrum of the CTS-GLY/KN after RBBR uptake (Fig. 3b) illustrates an outline similar to CTS-GLY/K with a slight shifting of some distinguished bands, evidencing that the adsorption of RBBR involves various functional groups of the CTS-GLY/KN.

The surface morphology of CTS-GLY/KN and CTS-GLY/KN after RBBR uptake, in addition to analysis of its chemical composition was done utilizing SEM-EDX analysis. The surface morphology of CTS-GLY/KN (Fig. 4a) shows a rugged surface with scarce porosities and crevices in existence. The EDX analysis highlights the existence of the following elements in the polymeric structure of CTS-GLY/KN: O, C, Al, N and P. After RBBR uptake onto the CTS-GLY/KN surface, the morphology of CTS-GLY/KN (Fig. 4b) looks more compact with 
less condensed porosities, in accordance with the RBBR uptake onto the surface of the CTSGLY/KN. The existence of $\mathrm{S}$ is displayed in the EDX analysis of CTS-GLY/KN after RBBR uptake that further confirms RBBR adsorption onto the surface of the CTS-GLY/KN.

\subsection{BBD model analysis}

BBD-RSM was utilized to study the interactive and individual impacts of the main adsorption variables on the $\mathrm{CR}$ and reduction of $\mathrm{COD}$ of $\mathrm{RBBR}$. In this study, the main parameters used were CTS-GLY/KN dose, solution $\mathrm{pH}$, and time. Furthermore, analysis of variance (ANOVA) was used for further investigating the statistical analysis of the experimental results collected regarding CR and COD reduction of RBBR (Table 4). The F-value for CR was found to be 43.07 (p-value $<0.0001$ ), whereas the F-value for COD was found to be 28.45 (pvalue of 0.0001 ) according to Table 4, revealing that the BBD model is statistically significant for CR and COD reduction [48]. Furthermore, the values of the coefficient of determination $\left(R^{2}\right)$ values are found to be 0.98 and 0.97 for CR and COD reduction, respectively, showing a high coherence between resulted and predicted values of $\mathrm{CR}$ and reduction of COD. Moreover, according to $\mathrm{BBD}$, the statistical values for $\mathrm{p}$-value are ought to be $<0.05$ to categorize a model

term in $\mathrm{BBD}$ as statistically significant, hence $\mathrm{A}, \mathrm{B}, \mathrm{C}, \mathrm{AB}, \mathrm{BC}$, and $\mathrm{C}^{2}$ model terms are considered statistically significant. The other model terms with $\mathrm{p}$-value $<0.05$ were disregarded in the second order polynomial equation; that is to obtain the best fit results for the model. Therefore, the correlation between the significant parameters towards CR and reduction of COD are shown in Eq. 5 and Eq. 6, respectively.

$$
\mathrm{CR}(\%)=+29.56+6.89 \mathrm{~A}-20.39 \mathrm{~B}+16.63 \mathrm{C}-6.05 \mathrm{AB}-12.03 \mathrm{BC}+13.55 \mathrm{C}^{2}
$$


COD reduction $(\%)=+14.98+10.34 \mathrm{~A}-13.59 \mathrm{~B}+14.63 \mathrm{C}-7.77 \mathrm{AB}-12.10 \mathrm{BC}+$

$19.07 C^{2}$

(6)

Another method that can also be implemented to validate the results of the BBD model is the graphical method. It works on investigating the nature of residuals distribution and the fitting of actual versus predicted values of $\mathrm{CR}$ and reduction of COD. Fig. 5a and Fig. 5b show the normal probability of residuals in the BBD model for $\mathrm{CR}$ and reduction of COD of RBBR, respectively. In the abovementioned figures, the close alignment of points into a linear form indicates the optimum normal distributions as well as the independence of residuals. Whereas Fig. 5c and Fig. 5d illustrate the correspondence between the predicted versus actual results of $\mathrm{CR}$ and COD reduction values. From the abovementioned figures, the actual and predicted values are relatively similar which can validate the BBD model statistically [49].

\subsection{The effect of adsorption operating parameters}

The significance of interaction amongst every set of two parameters on the CR and reduction of COD of RBBR was investigated. It was found that the effects of interaction between the CTS-GLY/KN dose (A), and $\mathrm{pH}$ (B) on the CR and reduction of COD of RBBR is statistically significant while the other parameter $($ time $=17.5 \mathrm{~min})$ is kept unchanged. The threedimensional response surface plots for the interaction between the CTS-GLY/KN dose and $\mathrm{pH}$ for the CR and reduction of COD are shown in Fig. 6a and Fig. 6b, respectively. From the figures, it can be deduced that decreasing the $\mathrm{pH}$ of the solution from 10 to 4 causes an increase in the $\mathrm{CR}(\%)$ and COD reduction (\%). In the same context, the $\mathrm{pH}_{\mathrm{pzc}}$ of the CTS-GLY/KN is determined to be 7.0 as presented in Fig. 6c. This result shows that negative charge can be 
acquired on the surface of CTS-GLY/KN at $\mathrm{pH}>\mathrm{pH}_{\mathrm{pzc}}$. Consequently, when solution $\mathrm{pH}$ reaches 4 the charge on the surface of CTS-GLY/KN can be converted to positive charge, signifying the ability of CTS-GLY/KN on adsorption of anionic RBBR dye. Thereby, an intense electrostatic attraction can take place between the surface functional groups of CTS-GLY/KN that are positively charged and the RBBR's anionic group as seen in Eq. 7:

$$
\mathrm{CTS}-\mathrm{GLY} / \mathrm{KN}-\mathrm{NH}_{3}^{+}+\mathrm{RBBR}^{-} \leftrightarrow \mathrm{CTS}-\mathrm{GLY} / \mathrm{KN}-\mathrm{NH}_{3}^{+} \ldots{ }^{-} \mathrm{RBBR}
$$

The interaction between $\mathrm{pH}(\mathrm{B})$ and time (C) on the $\mathrm{CR}$ and reduction of COD of RBBR is statistically significant while the other parameter (CTS-GLY/KN dose $=0.06 \mathrm{~g}$ ) is kept unchanged. Fig. 6d and Fig. 6e illustrate the three-dimensional response surface plots for the impact of the interaction between the two parameters of $\mathrm{pH}$ and time on the $\mathrm{CR}$ and reduction of COD, respectively. As shown in the abovementioned figures, the increase in the contact time from 5 to 30 minutes led to an increase in the $\mathrm{CR}(\%)$ and reduction of COD (\%) of RBBR from $12.5 \%$ to $97.3 \%$ and $5.6 \%$ to $81.8 \%$, respectively. This observation can be assigned to the extension of time for the molecules of RBBR dye to penetrate deeper into internal pores in the polymeric structure of the CTS-GLY/KN.

\subsection{Adsorption study}

In this study, the impact of the contact time on RBBR dye uptake onto the surface of CTS-GLY/KN was investigated. Different initial RBBR concentrations (50, 100, 150, 200, 300, and $400 \mathrm{mg} / \mathrm{L}$ ) in the solution were used for testing and validation of the results while the CTSGLY/KN dose $(0.06 \mathrm{~g} / 100 \mathrm{~mL})$, solution $\mathrm{pH}(4)$ and temperature $\left(30^{\circ} \mathrm{C}\right)$ were kept unchanged. Fig. 7a illustrates the breakthrough curves of adsorption capacities of CTS-GLY/KN, $q_{t}(\mathrm{mg} / \mathrm{g})$ 
versus time $(\mathrm{min})$ at various RBBR initial concentrations. As shown in Fig. 7a, the increase in concentration of RBBR starting from 50 gradually to $400 \mathrm{mg} / \mathrm{L}$ led to an increment in adsorption capacity of CTS-GLY/KN towards RBBR molecules from 58.6 to $284.9 \mathrm{mg} / \mathrm{g}$. This result corresponds with the greater concentration gradient which in return increments the diffusion of RBBR molecules in the internal pores of the CTS-GLY/KN leading to the RBBR molecules moving to active adsorption sites [50].

\subsection{Kinetic modeling}

To investigate the RBBR adsorption behavior on the CTS-GLY/KN surface, in addition to specifying the adsorption mechanism, kinetics of the RBBR adsorption on the surface of CTSGLY/KN were examined by applying two kinetic models: pseudo-first order (PFO) and pseudosecond order (PSO) [51, 52]. The non-linear formulas of pseudo-first order and pseudo-second order kinetic models are shown by the following Eq. 8 and Eq. 9, respectively.

$$
\begin{aligned}
& \left.q_{t}=q_{e^{(1-\exp }}^{-k_{1} t}\right) \\
& q_{t}=\frac{q_{e}^{2} k_{2} t}{1+q_{e} k_{2} t}
\end{aligned}
$$

where, $q_{t}$ and $q_{e}(\mathrm{mg} / \mathrm{g})$ represent the amount of RBBR adsorbed by CTS-GLY/KN at various time (t), and at equilibrium, respectively. $k_{1}\left(\mathrm{~min}^{-1}\right)$ represents the PFO rate constant while $k_{2}$ (g/mg min) represents the PSO rate constant. Table 5 summaries the kinetic parameters values for adsorption of RBBR on CTS-GLY/KN surface. The kinetic data of the RBRR adsorption (Table 5) on the CTS-GLY/KN surface demonstrates better compliance with PSO model in terms of the $R^{2}$ and calculated $q_{e}$ values compared to PFO model, exhibiting chemisorption 
phenomenon as the main factor in adsorption of RBBR on the surface of CTS-GLY/KN surface [53].

\subsection{Isotherm modeling}

One of the indispensable parameters to explain the interactions between RBBR molecules and the surface of CTS-GLY/KN is the equilibrium isotherm. Three widely used isotherms (Langmuir [54], Freundlich [55], and Temkin [56]) were selected to explain the RBBR-CTS-GLY/KN interactions, affinity of CTS-GLY/KN towards RBBR molecules, and determine the uptake capacity of CTS-GLY/KN for RBBR. The non-linear formulas of Langmuir, Freundlich, and Temkin isotherms are given in Eqs. 10-12, respectively.

$$
\begin{aligned}
& q_{e}=\frac{q_{\max } K_{a} C_{e}}{1+K_{a} C_{e}} \\
& q_{e}=K_{f} C_{e}^{1 / n} \\
& q_{e}=\frac{R T}{b_{T}} \ln \left(K_{T} C_{e}\right)
\end{aligned}
$$

While $C_{e}(\mathrm{mg} / \mathrm{L})$ represents the concentration of RBBR in solution at equilibrium, $q_{\max }(\mathrm{mg} / \mathrm{g})$ indicates the monolayer adsorption capacity of the CTS-GLY/KN, and $q_{e}(\mathrm{mg} / \mathrm{g})$ is the uptake value of RBBR at equilibrium concentration. $K_{a}(\mathrm{~L} / \mathrm{mg}), K_{f}(\mathrm{mg} / \mathrm{g})(\mathrm{L} / \mathrm{mg})^{1 / \mathrm{n}}$, and $K_{T}(\mathrm{~L} / \mathrm{mg})$ are Langmuir, Freundlich, and Temkin constants, respectively. $R(8.314 \mathrm{~J} / \mathrm{mol} \mathrm{K})$ represents the gas constant, $T(\mathrm{~K})$ is temperature, $b_{T}(\mathrm{~J} / \mathrm{mol})$ is the heat of adsorption and $n$ represents adsorption intensity. Fig. $7 \mathrm{~b}$ illustrates the non-linear isotherms' plots, and Table 6 presents the values of isotherm parameters. From the $R^{2}$ values of the isotherm models (Table 6), it can be derived that the adsorption of RBBR onto CTS-GLY/KN surface is more accurately explained by both 
Langmuir and Freundlich models, demonstrating monolayer and multilayer coverage of the RBBR dye on the surface of CTS-GLY/KN are occurred simultaneously [57]. The $q_{\max }$ of RBBR on the CTS-GLY/KN surface is determined to be $447.1 \mathrm{mg} / \mathrm{g}$ at $30{ }^{\circ} \mathrm{C}$. The $q_{\max }$ of RBBR in multiple different adsorbents including CTS-GLY/KN is compared in Table 7. According to Table 7, it could be derived that CTS-GLY/KN is an effective adsorbent to remove the reactive dyes as well as to reduce COD from aquatic media.

\subsection{Adsorption mechanism of RBBR}

The RBBR adsorption mechanism onto CTS-GLY/KN surface is suggested according to the existing surface functional groups on the CTS-GLY/KN surface, e.g. $-\mathrm{NH}_{2},-\mathrm{OH}$, and $\equiv \mathrm{SiOH}$. Fig. 8 summarizes the interactions possible between RBBR and CTS-GLY/KN. The highest RBBR dye removal was observed at $\mathrm{pH} 4$ where the surface of the CTS-GLY/KN is having positive charge. The cationic amino groups $\left(\mathrm{NH}_{3}{ }^{+}\right)$of CTS and positive charges of $\mathrm{KN}$ result into this positive charge on the surface of the CTS-GLY/KN, resulting into electrostatic attractions occurring between the positively charged CTS-GLY/KN surface and negative sulfonate groups $\left(-\mathrm{SO}_{3}{ }^{-}\right)$of $\mathrm{RBBR}$. Hydrogen bonding occurring between the free hydrogens of the CTS-GLY/KN with nitrogen and oxygen elements available in the structure of RBBR dye is the next significant adsorption mechanism of RBBR onto the surface of CTS-GLY/KN. Moreover, Yoshida H-bonding interaction can occur between hydrogen of hydroxyl groups of CTS-GLY/KN and the aromatic rings of RBBR dye as well [59]. Finally, n- $\pi$ interaction takes place between electron donating groups of oxygen and nitrogen on CTS-GLY/KN surface and $\pi$ system (electron acceptor) in the aromatic rings of RBBR. 


\section{Conclusions}

CTS-GLY/KN composite is successfully fabricated and employed for CR and reduction of COD of RBBR from aqueous solutions. The optimum conditions for $\mathrm{CR}$ and reduction of COD of RBBR were found to be $\mathrm{pH} 4$, dose $(0.06 \mathrm{~g})$, and time $(30 \mathrm{~min})$. The results found that the highest values of $\mathrm{CR}(97.3 \%)$ and COD reduction $(81.8 \%)$ of RBBR can be achieved by the following significant interactions: $\mathrm{AB}$ and $\mathrm{BC}$. The adsorption capacity obtained from Langmuir model reflected a high adsorption capacity $(447.1 \mathrm{mg} / \mathrm{g})$ of CTS-GLY/KN composite. The mechanism adsorption of RBBR dye by CTS-GLY/KN is attributed to electrostatic, $n-\pi$, Hydrogen bonding, and Yoshida H-bonding interactions. The results also reveal that CTSGLY/KN could be utilized as an adequate adsorbent for color removal and reduction of COD of textile dyes from aqueous environment.

\section{Acknowledgements}

The authors would like to thank the faculty of applied sciences for facilitating the research project. The fifth and sixth authors would like to thank Researchers Supporting Project No. (RSP-2020/138), King Saud University, Riyadh, Saudi Arabia.

\section{References}

[1] Rashid RA, Ishak MAM, Hello KM (2018) Adsorptive removal of methylene blue by commercial coconut shell activated carbon. Sci Lett 12:27-97.

[2] Rafaie R A, Yusop N F M, Azmi N F, Abdullah N S, Ramli N I R (2021) Photocatalytic degradation of methylene blue dye solution using different amount of $\mathrm{ZnO}$ as a photocatalyst, Sci. Lett. 15(1): 1-12. 
[3] Jawad, AH, Ishak MAM, Farhan AM, Ismail K (2017) Response surface methodology approach for optimization of color removal and COD reduction of methylene blue using microwave-induced $\mathrm{NaOH}$ activated carbon from biomass waste. Desalin Water Treat 62:208220.

[4] Wang M, He L, Wang M, Chen L, Yao S, Jiang W, Chen Y (2019) Simultaneous removal of $\mathrm{NH}_{3}-\mathrm{N}$ and COD from shale gas distillate via an integration of adsorption and photo-catalysis: A hybrid approach. J Environ Manage 249:109342.

[5] Yuan H, Chen L, Cao Z, Hong FF (2020) Enhanced decolourization efficiency of textile dye Reactive Blue 19 in a horizontal rotating reactor using strips of BNC-immobilized laccase: Optimization of conditions and comparison of decolourization efficiency. Biochem Eng $\mathrm{J}$ 156:107501.

[6] Nair V, Panigrahy A, Vinu R (2014) Development of novel chitosan-lignin composites for adsorption of dyes and metal ions from wastewater. Chem Eng J 254:491-502.

[7] Ada K, Ergene A, Tan S, Yalçın E (2009) Adsorption of Remazol Brilliant Blue R using ZnO fine powder: Equilibrium, kinetic and thermodynamic modeling studies. J Hazard. Mater 165(13):637-644.

[8] Zubair M, Manzar MS, Mu'azu ND, Anil I, Blaisi NI, Al-Harthi MA (2020) Functionalized MgAl-layered hydroxide intercalated date-palm biochar for Enhanced Uptake of Cationic dye: Kinetics, isotherm and thermodynamic studies. Appl Clay Sci 190:105587.

[9] You M, Li W, Pan Y, Fei P, Wang H, Zhang W, Meng J (2019) Preparation and characterization of antibacterial polyamine-based cyclophosphazene nanofiltration membranes. $\mathrm{J}$ Membr Sci 592:117371.

[10] El Hassani K, Kalnina D, Turks M, Beakou BH, Anouar A (2019) Enhanced degradation of an azo dye by catalytic ozonation over Ni-containing layered double hydroxide nanocatalyst. Sep Purif Technol 210:764-774.

[11] Mantasha I, Hussain S, Ahmad M, Shahid M (2020) Two dimensional (2D) molecular frameworks for rapid and selective adsorption of hazardous aromatic dyes from aqueous phase. Sep Purif Technol 238:116413.

[12] Priyadarshi R, Rhim JW (2020) Chitosan-based biodegradable functional films for food packaging applications. Innov Food Sci Emerg Technol 62:102346.

[13] da Silva PM, Camparotto NG, Lira KT, Picone CS, Prediger P (2020) Adsorptive removal of basic dye onto sustainable chitosan beads: Equilibrium, kinetics, stability, continuous-mode adsorption and mechanism. Sustain Chem Pharm 18:100318.

[14] Bakshia PS, Selvakumara D, Kadirvelub K, Kumara NS (2020) Chitosan as an environment friendly biomaterial - a review on recent modifications and applications, Int J Biol Macromol 150:1072-1083. 
[15] Xu X, Cheng Y, Wu X, Fan P, Song R (2020) La (III)-bentonite/chitosan composite: A new type adsorbent for rapid removal of phosphate from water bodies. Appl Clay Sci 190:105547.

[16] Li J, Cai J, Zhong L, Cheng H, Wang H, Ma Q (2019) Adsorption of reactive red 136 onto chitosan/montmorillonite intercalated composite from aqueous solution. Appl Clay Sci 167:9-22.

[17] Ghiorghita CA, Borchert KB, Vasiliu AL, Zaharia MM, Schwarz D, Mihai M (2020) Porous thiourea-grafted-chitosan hydrogels: Synthesis and sorption of toxic metal ions from contaminated waters. Colloids Surface A: Physicochem Eng Asp 607:125504.

[18] Jawad AH, Malek NNA, Abdulhameed AS, Razuan, R (2020) Synthesis of Magnetic Chitosan-Fly Ash/ $\mathrm{Fe}_{3} \mathrm{O}_{4}$ Composite for Adsorption of Reactive Orange 16 Dye: Optimization by Box-Behnken Design. J Polym Environ 28(3):1068-1082.

[19] Vakili M, Deng S, Li T, Wang W, Wang W, Yu G (2018) Novel crosslinked chitosan for enhanced adsorption of hexavalent chromium in acidic solution. Chem Eng J 347:782-790.

[20] Tahira I, Aslam Z, Abbas A, Monim-ul-Mehboob M, Ali S, Asghar A (2019) Adsorptive removal of acidic dye onto grafted chitosan: A plausible grafting and adsorption mechanism. Int J Biol Macromol 136:1209-1218.

[21] Mahmoud ME, El-Ghanam AM, Mohamed RHA, Saad SR (2020) Enhanced adsorption of Levofloxacin and Ceftriaxone antibiotics from water by assembled composite of nanotitanium oxide/chitosan/nano-bentonite. Mater Sci Eng C 108:110199.

[22] Abdulhameed AS, Mohammad AT, Jawad AH (2019) Application of response surface methodology for enhanced synthesis of chitosan tripolyphosphate/ $\mathrm{TiO}_{2}$ nanocomposite and adsorption of reactive orange 16 dye. J Clean Prod 232:43-56.

[23] Alhwaige AA, Ishida H, Qutubuddin S (2019) Poly (benzoxazine-f-chitosan) films: The role of aldehyde neighboring groups on chemical interaction of benzoxazine precursors with chitosan. Carbohydr Polym 209:122-129.

[24] Lyu H, Fan J, Ling Y, Yu Y, Xie Z (2019) Functionalized cross-linked chitosan with ionic liquid and highly efficient removal of azo dyes from aqueous solution. Int $\mathrm{J}$ Biol Macromol 126:1023-1029.

[25] Huang R, Liu Q, Huo J, Yang B (2017) Adsorption of methyl orange onto protonated crosslinked chitosan. Arab J Chem 10(1):24-32.

[26] Malek NNA, Jawad AH, Abdulhameed AS., Ismail K, Hameed BH (2020) New magnetic Schiff's base-chitosan-glyoxal/fly ash/ $\mathrm{Fe}_{3} \mathrm{O}_{4}$ biocomposite for the removal of anionic azo dye: An optimized process. Int J Biol Macromol 146:530-539.

[27] Zhu HY, Jiang R, Xiao L (2010) Adsorption of an anionic azo dye by chitosan $/ \mathrm{kaolin} / \gamma$ $\mathrm{Fe}_{2} \mathrm{O}_{3}$ composites. Appl Clay Sci 48(3):522-526.

[28] Anwar Y (2018) Antibacterial and lead ions adsorption characteristics of chitosanmanganese dioxide bionanocomposite. Int. J. Biol. Macromol. 111:1140-1145. 
[29] Kameda T, Honda R, Kumagai S, Saito Y, Yoshioka T (2019) Uptake of heavy metal cations by chitosan-modified montmorillonite: Kinetics and equilibrium studies. Mater Chem Phys 236:121784.

[30] Alhwaige AA, Ishida H, Qutubuddin S (2016) Carbon aerogels with excellent CO2 adsorption capacity synthesized from clay-reinforced biobased chitosan-polybenzoxazine nanocomposites. ACS Sustain Chem Eng 4(3), 1286-1295.

[31] Alawady AR, Alshahrani AA, Aouak TA, Alandis NM (2020) Polysulfone membranes with CNTs/Chitosan biopolymer nanocomposite as selective layer for remarkable heavy metal ions rejection capacity. Chem Eng J 388:124267.

[32] Jabli M (2020) Synthesis, characterization, and assessment of cationic and anionic dye adsorption performance of functionalized silica immobilized chitosan bio-polymer. Int J Biol Macromol 153:305-316.

[33] Yavuz Ö, Saka C (2013) Surface modification with cold plasma application on kaolin and its effects on the adsorption of methylene blue. Appl Clay Sci 85:96-102.

[34] Rekik SB, Gassara S, Bouaziz J, Deratani A, Baklouti S, (2017) Development and characterization of porous membranes based on kaolin/chitosan composite. Appl Clay Sci 143:19.

[35] Leiviskä T, Sarpola A, Tanskanen J (2012) Removal of lipophilic extractives from debarking wastewater by adsorption on kaolin or enhanced coagulation with chitosan and kaolin. Appl clay Sci 61:22-28.

[36] Liu B, Zheng H, Wang Y, Chen X, Zhao C, An Y, Tang X (2018) A novel carboxyl-rich chitosan-based polymer and its application for clay flocculation and cationic dye removal. Sci Total Environ 640:107-115.

[37] Banu HAT, Karthikeyan P, Vigneshwaran S, Meenakshi S (2020) Adsorptive performance of lanthanum encapsulated biopolymer chitosan-kaolin clay hybrid composite for the recovery of nitrate and phosphate from water. Int J Biol. Macromol 154:188-197.

[38] Mohammad AT, Abdulhameed AS, Jawad AH (2019) Box-Behnken design to optimize the synthesis of new crosslinked chitosan-glyoxal/ $\mathrm{TiO}_{2}$ nanocomposite: Methyl orange adsorption and mechanism studies. Int J Boil Macromol 129:98-109.

[39] Dalvand A, Nabizadeh R, Ganjali MR, Khoobi M, Nazmara S, Mahvi AH (2016) Modeling of Reactive Blue 19 azo dye removal from colored textile wastewater using L-argininefunctionalized $\mathrm{Fe}_{3} \mathrm{O}_{4}$ nanoparticles: Optimization, reusability, kinetic and equilibrium studies. J Magn Magn Mater 404:179-189.

[40] Vieira RS, Beppu MM (2006) Interaction of natural and crosslinked chitosan membranes with Hg (II) ions. Colloids Surf A Physicochem Eng Asp 279 (1-3):196-207.

[41] Jawad AH, Abdulhameed AS, Malek NNA, ALOthman ZA (2020) Statistical optimization and modeling for color removal and COD reduction of reactive blue 19 dye by mesoporous chitosan-epichlorohydrin/kaolin clay composite. Int J Boil Macromol 164:4218-4230. 
[42] Jawad AH, Abdulhameed AS (2020) Facile synthesis of crosslinked chitosantripolyphosphate/kaolin clay composite for decolourization and COD reduction of remazol brilliant blue R dye: Optimization by using response surface methodology. Colloids Surf A Physicochem Eng Asp 605:125329.

[43] Sing KS (1985) Reporting physisorption data for gas/solid systems with special reference to the determination of surface area and porosity (Recommendations 1984), Pure Appl Chem 57(4):603-619.

[44] Jawad AH, Abdulhameed AS (2020) Mesoporous Iraqi red kaolin clay as an efficient adsorbent for methylene blue dye: Adsorption kinetic, isotherm and mechanism study. Surf Interfac 18:100422.

[45] Maity J, Ray SK (2018) Chitosan based nano composite adsorbent-synthesis, characterization and application for adsorption of binary mixtures of $\mathrm{Pb}$ (II) and $\mathrm{Cd}$ (II) from water. Carbohydr Polym 182:159-171.

[46] Alhwaige AA, Ishida H, Qutubuddin S (2020) Chitosan/polybenzoxazine/clay mixed matrix composite aerogels: preparation, physical properties, and water absorbency. Appl Clay Sci 184:105403.

[47] Vardikar HS, Bhanvase BA, Rathod AP, Sonawane SH (2018) Sonochemical synthesis, characterization and sorption study of Kaolin-Chitosan- $\mathrm{TiO}_{2}$ ternary nanocomposite: Advantage over conventional method. Mater Chem Phys 217:457-467.

[48] Khajeh M, Ghaemi A (2020) Exploiting response surface methodology for experimental modeling and optimization of $\mathrm{CO}_{2}$ adsorption onto $\mathrm{NaOH}$-modified nanoclay montmorillonite. $\mathrm{J}$ Environ Chem Eng 8:103663.

[49] Abdulhameed AS, Jawad AH, Mohammad AT (2019) Synthesis of chitosan-ethylene glycol diglycidyl ether/ $/ \mathrm{TiO}_{2}$ nanoparticles for adsorption of reactive orange 16 dye using a response surface methodology approach. Bioresour Technol 293:122071.

[50] Jawad AH, Abdulhameed AS, Mastuli MS (2020) Acid-factionalized biomass material for methylene blue dye removal: a comprehensive adsorption and mechanism study. J Taibah Univ Sci 14(1):305-313.

[51] Lagergren S (1898) Zur theorie der sogenannten adsorption geloster stoffe, Vet.Akad.Handl. 24:1-39.

[52] Ho YS, McKay G (1998) Sorption of dye from aqueous solution by peat, Chem Eng J 70:115-124.

[53] Lu F, Dong A, Ding G, Xu K, Li J, You L (2019) Magnetic porous polymer composite for high performance adsorption of acid red 18 based on melamine resin and chitosan. J Mol Liq 294:111515.

[54] Langmuir I (1918) The adsorption of gases on plane surfaces of glass, mica and platinum. J Am Chem Soc 40:1361-1403. 
[55] Frenudlich HMF (1906) Over the adsorption in solution. J Phys Chem 57:385-471.

[56] Temkin MI (1940) Kinetics of ammonia synthesis on promoted iron catalysts, Acta physiochim URSS 12:327-356.

[57] Prakash S, Kumar M, Tripathi BP, Shahi VK (2010) Sol-gel derived poly (vinyl alcohol)-3(2-aminoethylamino) propyl trimethoxysilane: cross-linked organic-inorganic hybrid beads for the removal of $\mathrm{Pb}$ (II) from aqueous solution. Chem Eng J 162(1):28-36.

[58] Janaki V, Oh BT, Shanthi K, Lee KJ, Ramasamy AK, Kamala-Kannan S (2012) Polyaniline/chitosan composite: an eco-friendly polymer for enhanced removal of dyes from aqueous solution. Synth Met 162(11-12):974-980.

[59] Li X, Sun C, Cao W, Hu C, Zhao Y (2019) Comparative adsorption of remazol brilliant blue $\mathrm{R}$ and copper in aqueous solutions by carbon nanotubes with different levels of carboxyl group and specific surface area. Mater Res Express 6(10):1050e2.

[60] Iqbal M, Saeed A (2007) Biosorption of reactive dye by loofa sponge-immobilized fungal biomass of Phanerochaete chrysosporium. Process Biochem 42(7):1160-1164.

[61] Monsef Khoshhesab Z, Modaresnia N (2019) Adsorption of Acid Black 210 and Remazol Brilliant Blue R onto magnetite nanoparticles. Inorg Nano-Met Chem 49(8):231-239.

[62] Rahmat NA, Ali AA, Hussain N, Muhamad MS, Kristanti RA, Hadibarata T (2016) Removal of Remazol Brilliant Blue R from aqueous solution by adsorption using pineapple leaf powder and lime peel powder. Water Air Soil Pollut 227(4):105.

[63] Bedin KC, de Azevedo, SP, Leandro PK, Cazetta AL, Almeida VC (2017) Bone char prepared by $\mathrm{CO}_{2}$ atmosphere: Preparation optimization and adsorption studies of Remazol Brilliant Blue R. J Clean Prod 161:288-298.

[64] Jawad AH, Mohammed IA, Abdulhameed AS (2020) Tuning of Fly Ash Loading into Chitosan-Ethylene Glycol Diglycidyl Ether Composite for Enhanced Removal of Reactive Red 120 Dye: Optimization Using the Box-Behnken Design. J Polym Environ 28(10):2720-2733. 
Figures

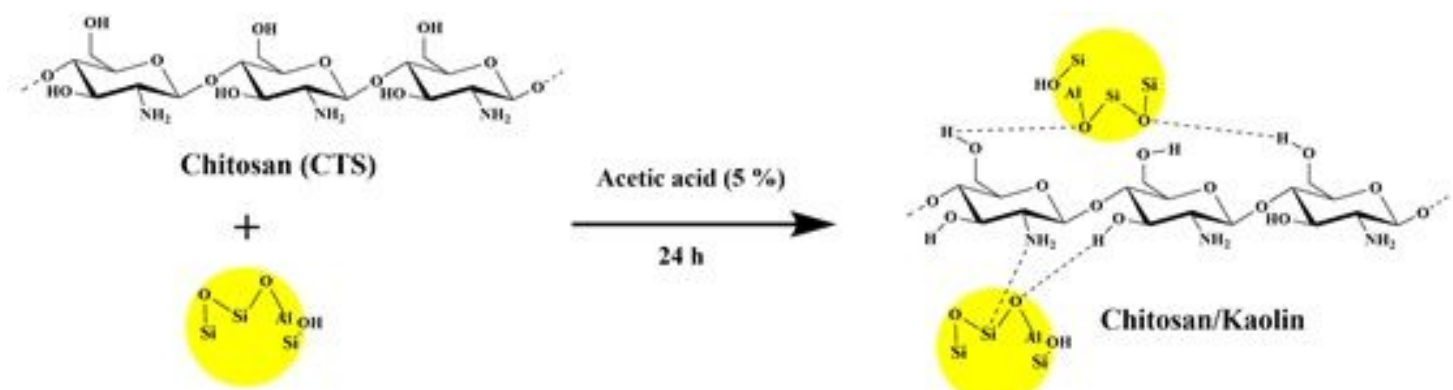

Kaolin (KN)
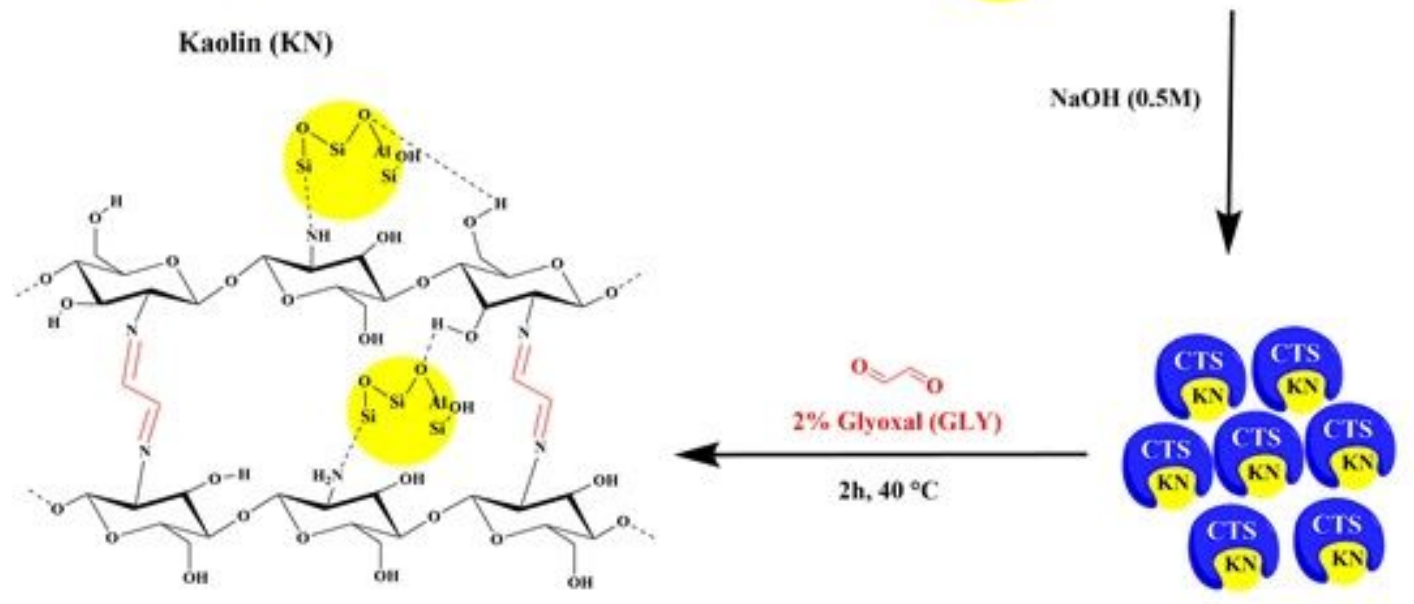

Chitosan-glyoxal/kaolin composite (CTS-GLY/KN)

Chitosan/Kaolin beads (CTS/KN)

Figure 1

Synthesis steps of CTS-GLY/KN

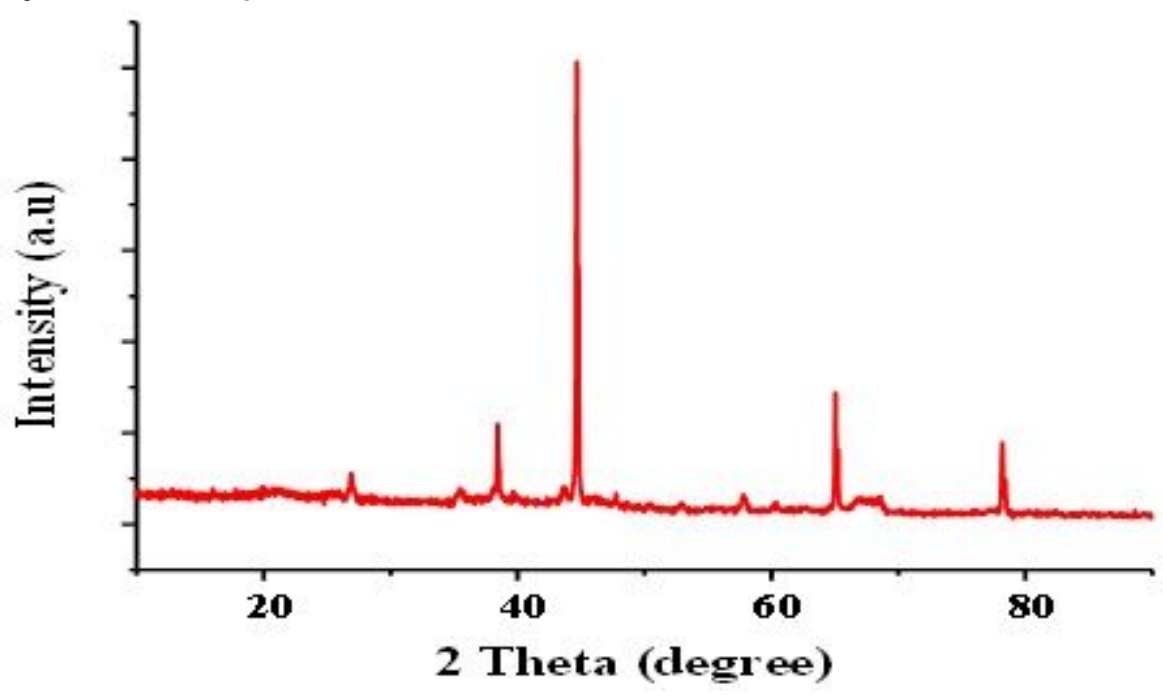

Figure 2

D pattern of CTS-GLY/KN 


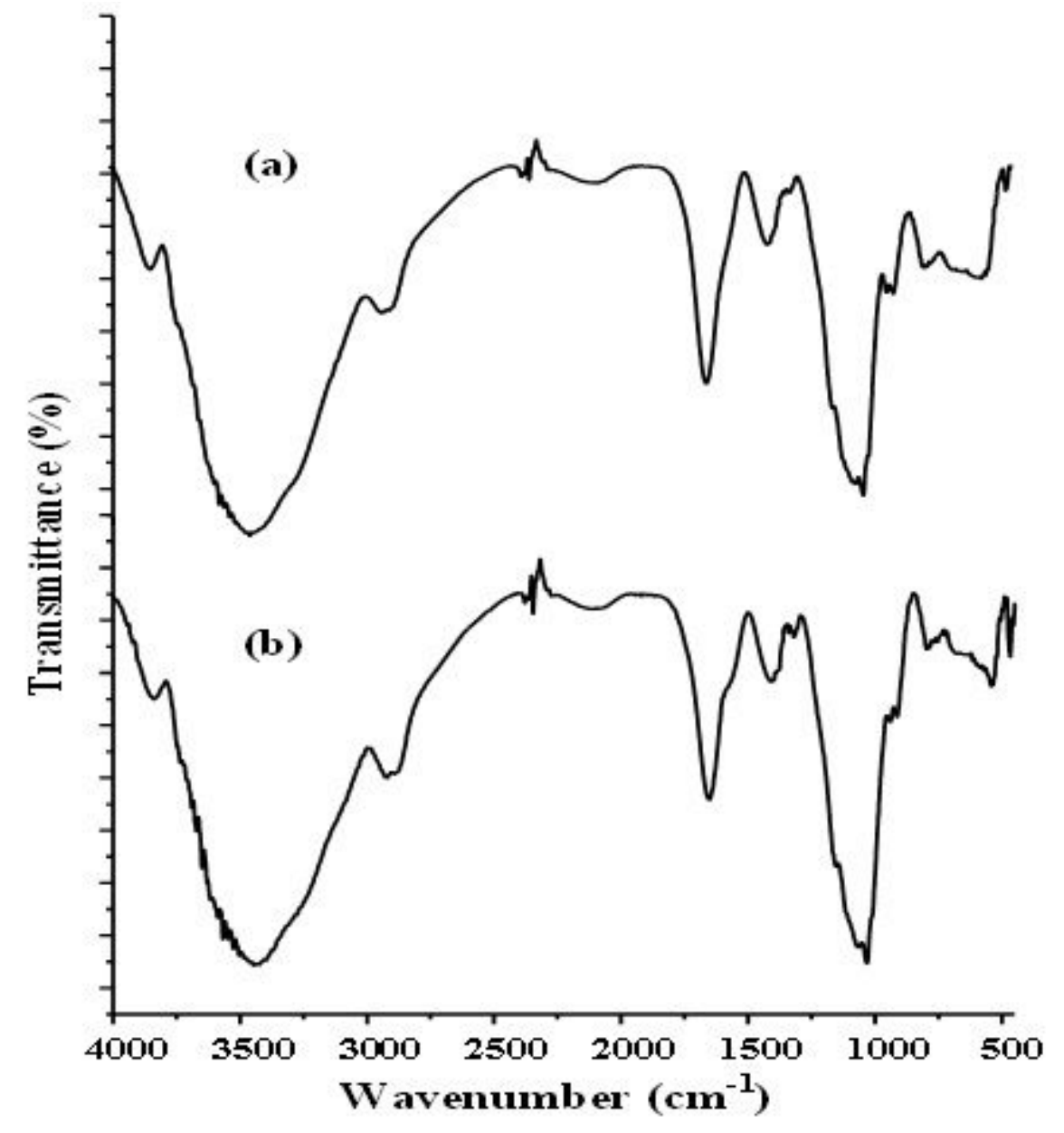

Figure 3

FTIR spectra of (a) CTS-GLY/KN, and (b)CTS-GLY/KN after adsorption of RBBR 

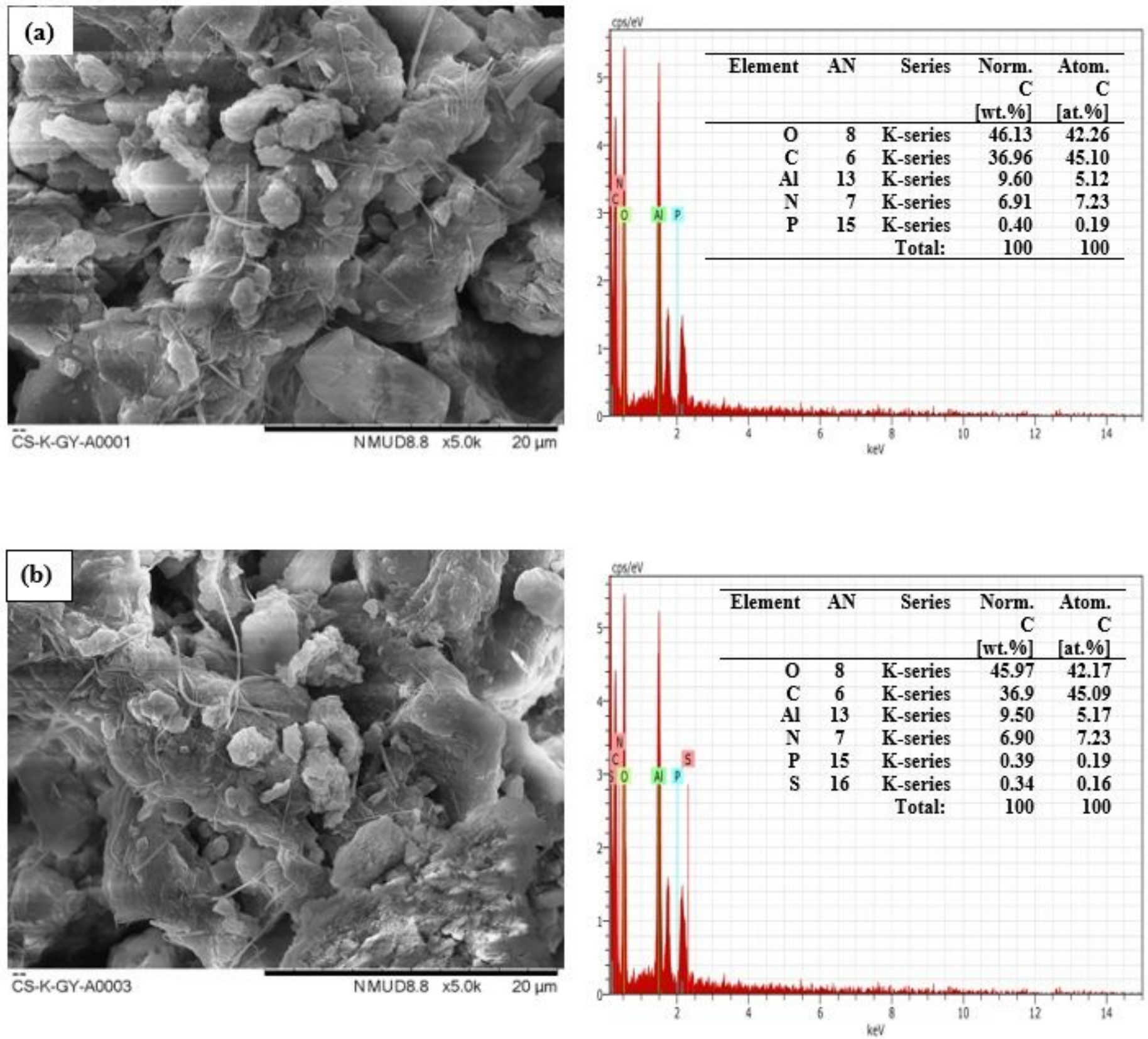

Figure 4

SEM images and EDX spectra of (a) CTS-GLY/KN, and (b) CTS-GLY/KN after adsorption of RBBR at magnification power 5000x. 

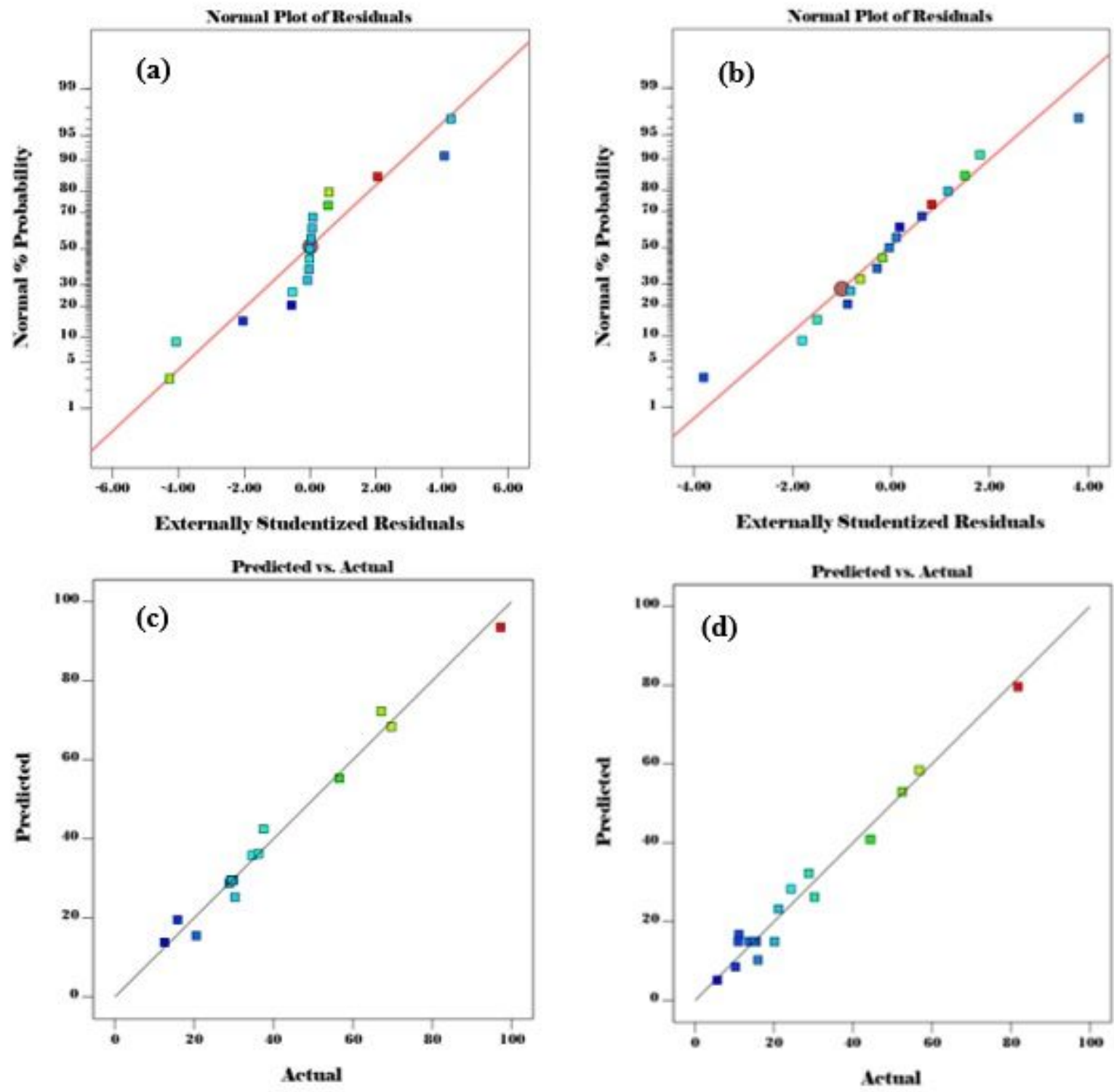

\section{Figure 5}

Normal probability plot of residuals for (a) RBBR color removal and (b) COD reduction, plot of the relationship between the predicted and actual values of (c) RBBR color removal and (d) COD reduction 

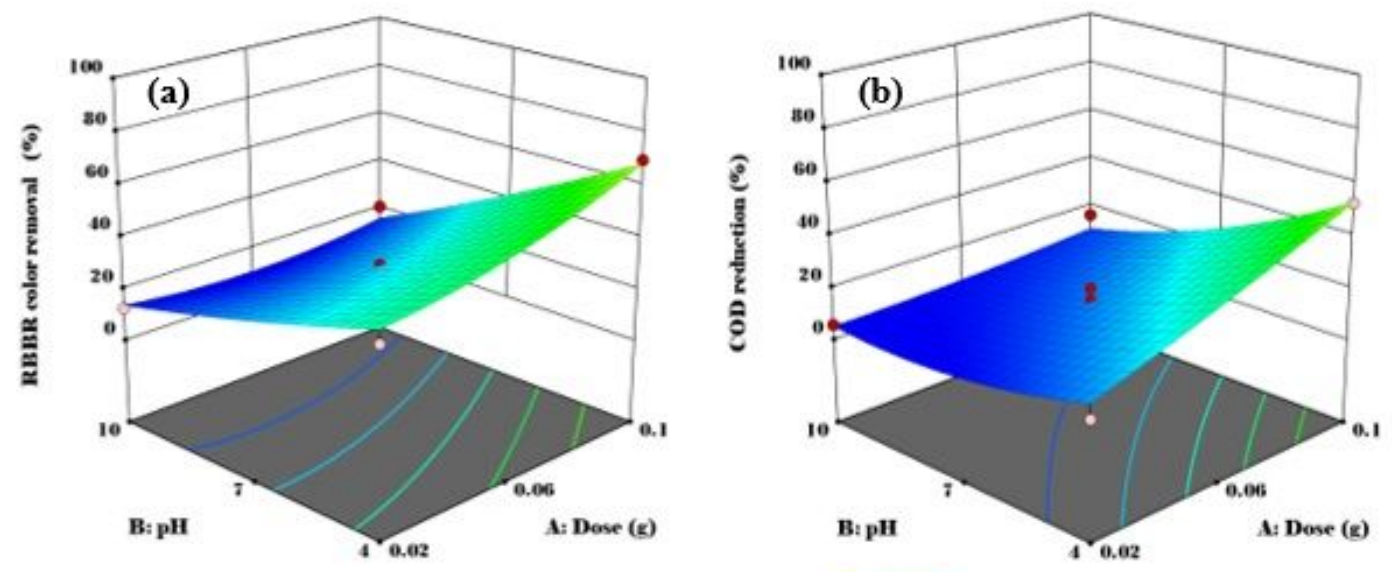

12.5 97.3

5.6

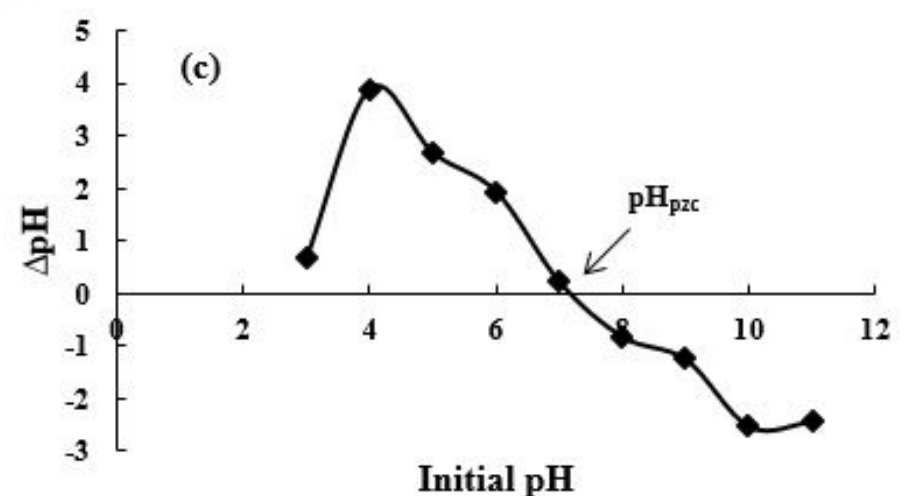

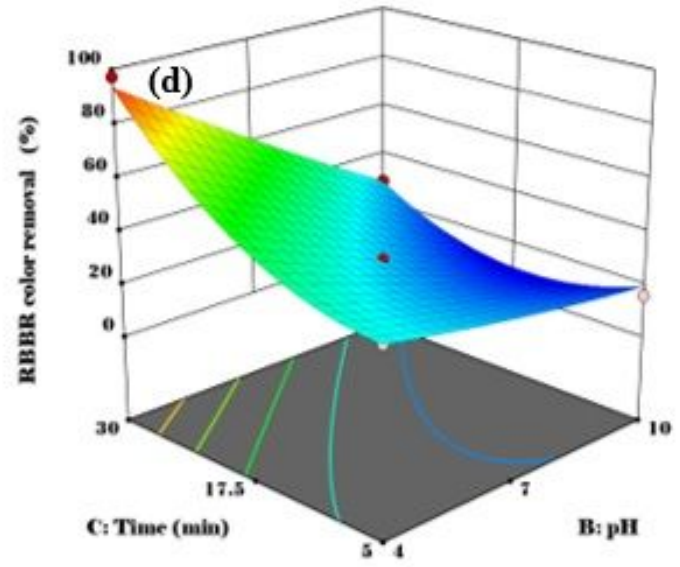

12.5

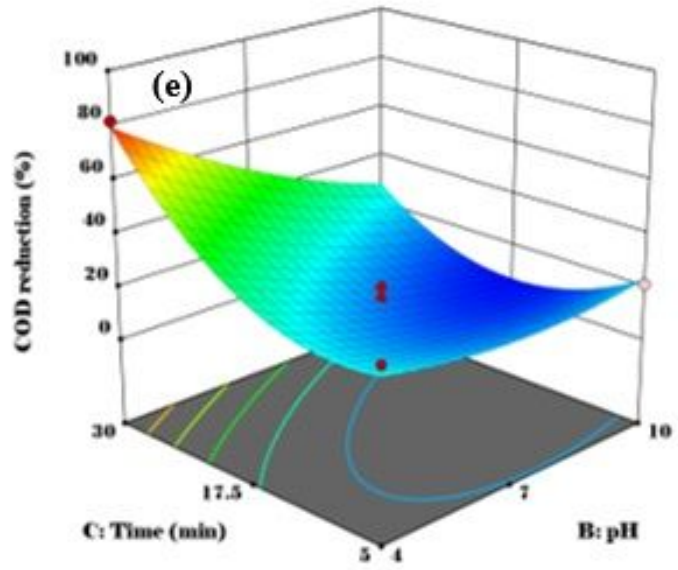

$\begin{array}{ll}5.6 \longrightarrow 81.8 & \end{array}$

\section{Figure 6}

3D response surfaces plots of significant interactions on RBBR color removal and COD reduction: (a) and (b) for AB interaction; (d) and (e) for BC interaction; whereas (c) pHpzc of CTS-GLY/KN. 

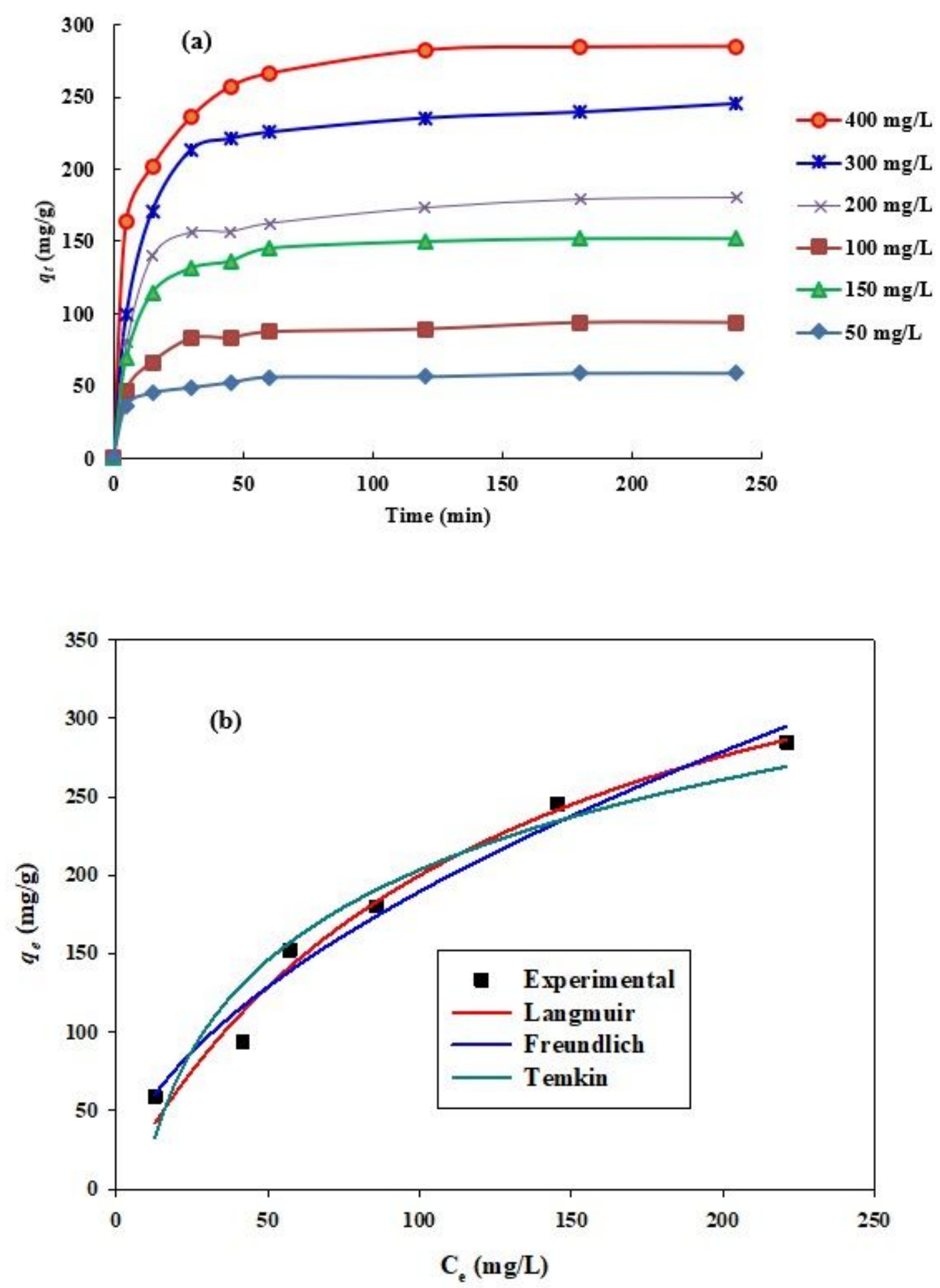

\section{Figure 7}

(a) Effect of the contact time on RBBR adsorption at different initial concentrations and (b) adsorption isotherms of RBBR by CTS-GLY $/ \mathrm{KN}$ (dose $=0.06 \mathrm{~g}$, $\mathrm{pH}$ of solution $=4$, temperature $=30 \mathrm{oC}$, agitation speed $=100$ strokes $/ \mathrm{min}$, and volume of solution $=100 \mathrm{~mL}$ ). 


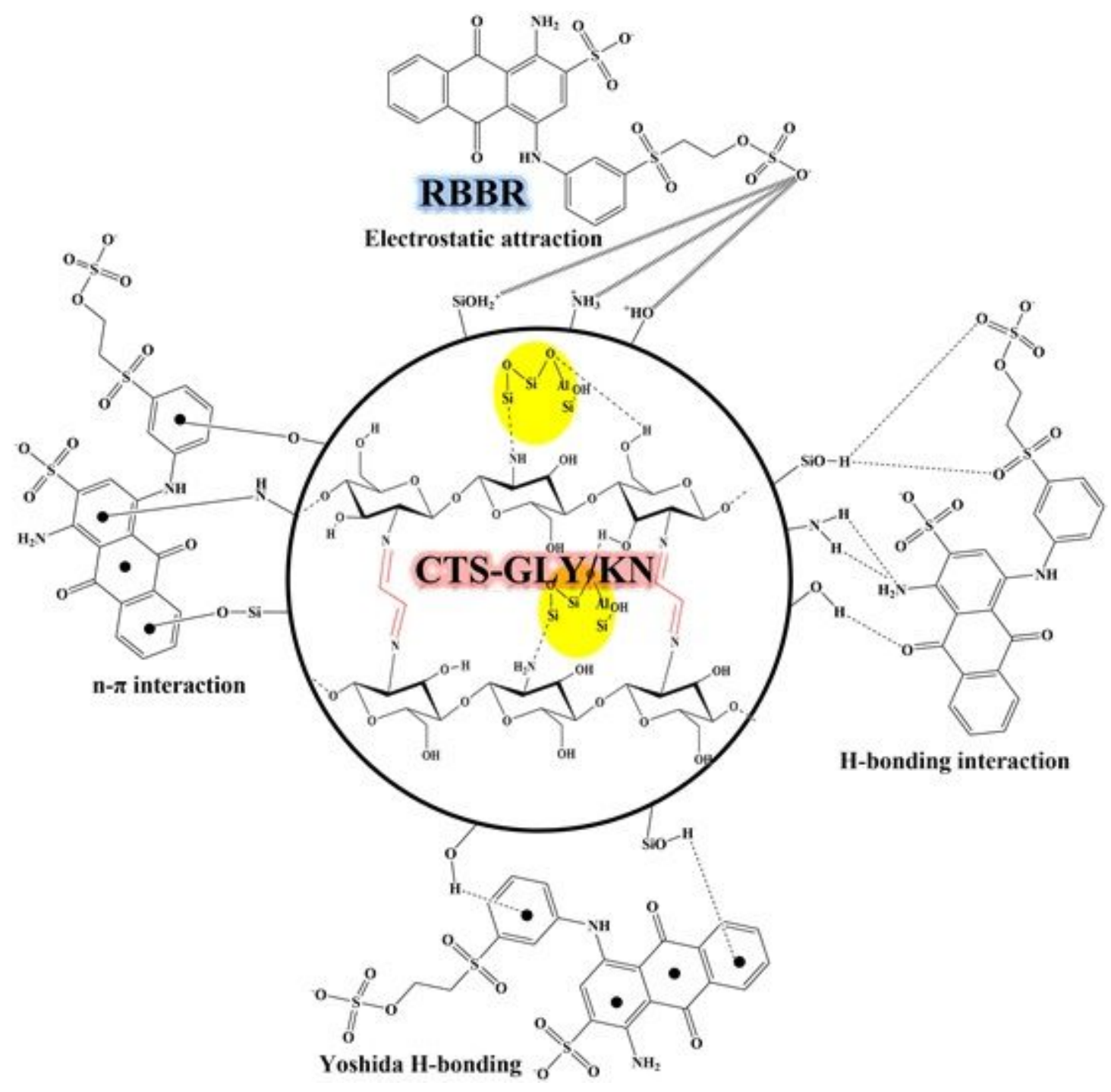

\section{Figure 8}

Illustration of the possible interaction between CTS-GLY/KN surface and RBBR including electrostatic attraction, hydrogen bonding interactions, Yoshida H-bonding, and $\pi-\pi$ interactions. 